\title{
Opportunistic round robin scheduling for V-BLAST systems over multiuser MIMO channels
}

\author{
Samir Al-Ghadhban
}

\begin{abstract}
In this paper, we study opportunistic round robin (ORR) uplink scheduling for vertical Bell Labs layered space-time architecture (V-BLAST) systems over multiuser multiple-input multiple-output (MIMO) channels. The proposed ORR scheduling method is compared to greedy scheduling. In greedy scheduling, the base station selects the best user based on a certain criterion without any consideration for fairness. On the other hand, ORR scheduling guarantees full fairness and each user will be served by excluding the previous selected users from the competition in the next round. The selected user spatially multiplexes his data over the transmit antennas. This spatial multiplexing (SM) scheme provides high data rates, while multiuser diversity obtained from scheduling improves the performance of the uplink system. The results show the performance and capacity gains obtained by scheduling. The greedy scheduler captures full multiuser diversity. In contrast, the ORR scheduler provides substantial signal-to-noise ratio (SNR) gains compared to round robin while guaranteeing full fairness to all users.
\end{abstract}

Keywords: Opportunistic round robin scheduling; MIMO multiuser uplink scheduling; VBLAST; Spatial multiplexing

\section{Introduction}

In multiuser multiple-input multiple-output (MIMO) wireless systems, optimizing the physical layer for each user does not necessarily optimize system performance nor takes advantage of statistical independence of fading channels for different users. Furthermore, users have different needs in terms of data rates, power constraints, and quality of service (QoS). These requirements make scheduling an important technique for optimizing the performance of communication systems and utilizing system resources efficiently. The wireless fading channels of users are usually independent. Thus, scheduling transmission to best users leads to a form of selection diversity known as multiuser selection diversity. In general, schedulers are designed to maximize system throughput and capacity or to minimize error rates. However, they should also provide fairness to users and minimize packet delays.

In single-input single-output (SISO) systems, where the base station and each mobile have one antenna, it has been shown that selecting the user who has the maximum

Correspondence: Samir@kfupm.edu.sa

Department of Electrical Engineering, King Fahd University of Petroleum and Minerals, Dhahran 31261, Saudi Arabia signal-to-noise ratio (MaxSNR) maximizes the total information capacity of uplink systems [1]. Similar results are also found for downlink systems from the base station to the mobile unit [2]. This selection criterion is known as MaxSNR.

For multiuser MIMO channels, most of the studies are based on theoretical information capacity and on the downlink [3-6], which is the broadcast channel from the base station to the mobile unit. Furthermore, it has been shown in [7] that space-time block coding (STBC) and scheduling are not a good match. In fact, scheduling to a user with a single antenna outperforms scheduling using STBC. The reason is that STBC averages the fades, while scheduling tends to benefit from high peaks in the fading channel. In addition, multiuser diversity obtained from scheduling is much higher than the spatial diversity of STBC. Therefore, STBC inherent diversity does not add much benefits. On the other hand, spatial multiplexing (SM) schemes match perfectly with scheduling since they provide high data rates while scheduling provides multiuser selection diversity.

\section{照 Springer}

(C) 2014 Al-Ghadhban; licensee Springer. This is an Open Access article distributed under the terms of the Creative Commons Attribution License (http://creativecommons.org/licenses/by/2.0), which permits unrestricted use, distribution, and reproduction in any medium, provided the original work is properly credited. 
In addition, for MIMO systems, scheduling could be done to a single user or multiple users. Scheduling to multiple users, i.e., allowing more than one user to transmit or receive at the same time, is optimal in terms of maximizing system capacity and throughput $[3,4,8]$. That is because the degrees of freedom provided by the multiuser MIMO system are much larger than those of the single user selection case. In [3], downlink scheduling to multiple users improved the average throughput compared to a single user scheduling. Furthermore, the authors in [8] showed that the optimal scheduler should allocate all power to at most $M_{\mathrm{R}}$ users, where $M_{\mathrm{R}}$ is the number of receive antennas at the base station. Also, they found that the optimal power resource allocation is water-filling in space and time. In [4], the authors found that multiuser scheduling reduces average delays experienced by users compared to a single-user scheduling.

For spatially multiplexed systems, greedy multiuser scheduling over MIMO systems was studied in [9], where we proposed and compared the performance of several user selection criteria for uplink vertical Bell Labs layered space-time architecture (V-BLAST) systems. Later in [10], the authors investigated user selection criteria that minimized the pairwise error performance for optimal and suboptimal lattice reduction (LR)-based MIMO detectors. In addition, a low-complexity user selection scheme with an iterative lattice reduction algorithm was proposed in [11], where the authors showed that their proposed algorithm provided comparable performance with the combinatorial approaches with much lower complexity. The effect of imperfect channel state information (CSI) was investigated in [12], where they showed that their proposed robust multiuser MIMO scheduling improved the system average throughput significantly.

To overcome the drawback of the greedy scheduler in [9] and to guarantee full fairness to all users, we propose in this paper to use opportunistic round robin (ORR) scheduling. In addition, we compare the performance of greedy and ORR scheduling schemes. We demonstrate the fundamental trade-off between performance and fairness in multiuser scheduling. The greedy scheme selects the best user without considering fairness among users. On the other hand, the ORR algorithm selects the best user first based on a scheduling criterion, then this selected user will be excluded from the search in the next round until all users are served. The result of this work shows that the ORR scheduler provides SNR gains compared to round robin scheduling while still providing full fairness to all users.

\section{System model}

We consider an uplink wireless communication system between $K$ users and a base station. Each user is equipped with $M_{\mathrm{T}}$ transmit antennas, and the receiver has $M_{\mathrm{R}}$ receive antennas $\left(M_{\mathrm{R}} \geq M_{\mathrm{T}}\right)$, as shown in Figure 1. The average SNR is assumed to be the same for all users by using power control. The MIMO channel is assumed to be an independent Rayleigh flat fading MIMO channel where each coefficient is an i.i.d complex Gaussian random variable with zero mean and unit variance.

In this paper, the base station compares the MIMO channels of all users and selects the best user one at a time based on a certain criterion. In general, for MIMO multiuser scheduling, the best set of transmit antennas could be selected, and this set might belong to more than one user. However, this approach requires more feedback and synchronization than a single user selection constraint. In addition, for MIMO multiuser scheduling, the scheduler should select the best set of $M_{\mathrm{T}}$ transmit antennas out of $K M_{\mathrm{T}}$ antennas. Thus, the search space will be huge, and suboptimal search algorithms should be proposed. However, this is out of the scope of this paper where we are focusing on analyzing and comparing the performance of user selection criteria.

Assuming that the base station has selected user $k$, the received signal from user $k$ will be

$$
\mathbf{y}_{k}=\mathbf{H}_{k} \mathbf{x}_{k}+\eta_{k}
$$

where $\mathbf{y}_{k}$ is an $M_{\mathrm{R}} \times 1$ received vector, $\mathbf{H}_{k}$ is an $M_{\mathrm{R}} \times M_{\mathrm{T}}$ MIMO channel matrix for the $k$ th user, $\mathbf{x}_{k}$ is an $M_{\mathrm{T}} \times 1$ transmitted symbols from user $k$, and $\eta_{k}$ is an $M_{\mathrm{R}} \times 1$ i.i.d complex AWGN vector of zero mean and variance $N_{0} / 2$ per dimension.

Channel state information (CSI) is assumed to be known only at the receiver (base station) for all users. Based on a selection criterion, the receiver compares all users and selects the best user at that time. Then, it informs the best user to transmit through a feedback channel.

\section{Scheduling schemes}

We compare in this study two scheduling schemes. They are greedy and opportunistic round robin. The greedy scheduler selects the best user based on a user selection criterion. This scheduler does not guarantee fairness in the sense that users with weak channel conditions will not be served. However, if all users have same channel statistics and strict power control is applied, then greedy scheduling will be fair on average. On the other hand, the ORR scheduler guarantees fair scheduling to all users. It selects the best user first based on a scheduling criterion. In the next round, this selected user is excluded from the search and only the remaining users are considered. This procedure is repeated until all users are served. 


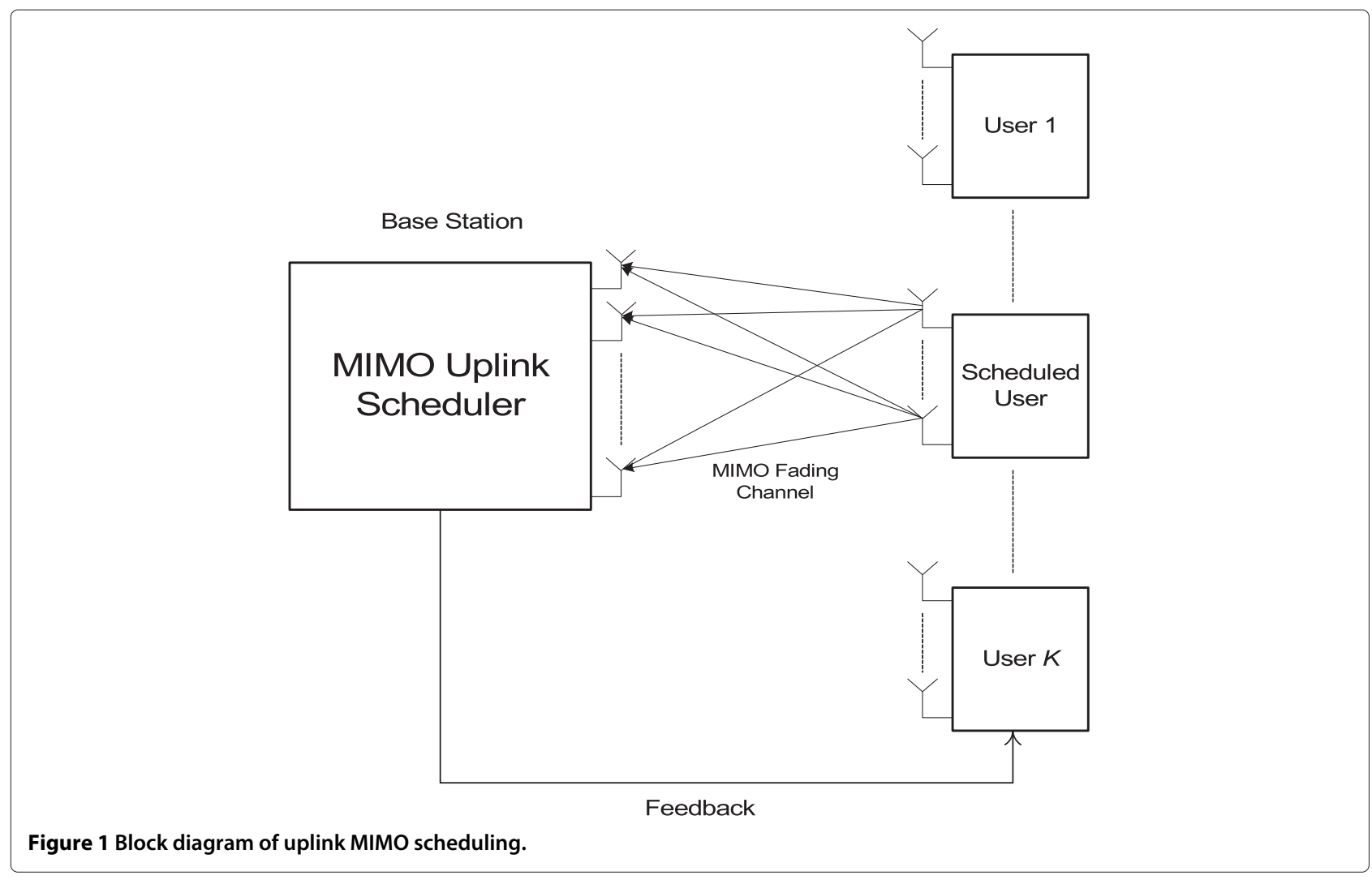

\section{Optimal MIMO user selection criteria}

Assuming that an optimal MIMO encoder/decoder is available, the optimal MIMO scheduler selects a user who has a channel matrix that maximizes the MIMO capacity:

$$
\begin{aligned}
C_{\max } & =\max _{k=1,2, \ldots, K} C_{k} ; \text { where } \\
C_{k} & =\log _{2}\left(\operatorname{det}\left(\mathbf{I}_{M_{\mathrm{R}}}+\frac{\mathrm{SNR}}{M_{\mathrm{T}}} \mathbf{H}_{k} \mathbf{H}_{k}^{H}\right)\right)
\end{aligned}
$$

where $\mathbf{I}_{M_{\mathrm{R}}}$ is the identity matrix and $\mathbf{A}^{H}$ is the conjugate transpose (Hermitian) of A. Assuming that $M_{\mathrm{T}} \leq M_{\mathrm{R}}$, we can write

$$
C_{k}=\sum_{n=1}^{M_{\mathrm{T}}} \log _{2}\left(1+\frac{\mathrm{SNR}}{M_{\mathrm{T}}} \lambda_{n}\left(\mathbf{H}_{k}\right)\right)
$$

where $\lambda_{n}\left(\mathbf{H}_{k}\right)$ is the $n$th eigenvalue of $\mathbf{H}_{k} \mathbf{H}_{k}^{H}$. The maximum capacity is achieved when the channel is orthogonal [13], for which $\mathbf{H}_{k} \mathbf{H}_{k}^{H}$ is a diagonal martix with $\left\|\mathbf{H}_{k, n}\right\|_{F}^{2}$ as its $(n, n)$-th element, where $\left\|\mathbf{H}_{k, n}\right\|_{F}^{2}$ the squared Frobenius norm of the $n$th row of $\mathbf{H}_{k}$. In this case, $\lambda_{n}\left(\mathbf{H}_{k}\right)=$ $\left\|\mathbf{H}_{k, n}\right\|_{F}^{2}$. If the eigenvalues are equal, the resulting MIMO capacity will be

$$
\left.C_{k}=M_{\mathrm{T}} \log _{2}\left(1+\frac{\mathrm{SNR}}{M_{\mathrm{T}}}\left\|\mathbf{H}_{k, 1}\right\|_{F}^{2}\right)\right) .
$$

\section{V-BLAST user selection criteria}

V-BLAST [14] is a practical MIMO architecture that spatially multiplexes transmitted data over multiple transmit antennas. Data transmitted from each antenna is called a layer of information. At the receiver, a serial interference nulling and cancellation algorithm is used to detect each layer. Although V-BLAST is a full spatial multiplexing scheme, it has poor energy performance because of the lack of spatial diversity. The diversity order of V-BLAST is $M_{\mathrm{R}}-M_{\mathrm{T}}+1$ [15]. Thus, when the number of receive and transmit antennas is equal, there will be no spatial diversity. Therefore, V-BLAST makes a good match with scheduling since multiuser diversity will improve the system performance significantly.

V-BLAST applies successive nulling and cancellation algorithm to detect the spatially multiplexed data. The nulling part could be done by zero forcing (ZF) or minimum mean squared error (MMSE). For a single user system, the authors in [15] showed that both ZF and MMSE provide the same spatial diversity order. However, MMSE provides SNR gains compared to ZF. We investigate in this paper the effect of ZF and MMSE nulling matrices on multiuser systems with scheduling. As will be shown in the 'Simulation Results' section, the SNR gains provided by MMSE diminishes with multiuser selection diversity. 
This section presents user selection criteria for uplink V-BLAST users. It has been shown that in single-antenna systems, selecting the user that has the maximum SNR (MaxSNR) is optimal [1] and it maximizes the system information capacity. However, the MaxSNR scheduler is not optimal for V-BLAST users. The reason is that, unlike SISO systems, the received SNR (trace $\left(\mathbf{H}_{k} \mathbf{H}_{k}^{H}\right)$ ) does not reflect directly into the capacity of MIMO systems. Moreover, scheduling based on maximization of MIMO channel capacity as in (3) is also not optimal for V-BLAST since it uses suboptimal detection algorithm. Since V-BLAST is an open loop system and all layers have the same rate, an outage in capacity will occur if an outage happens in at least one layer. Therefore, V-BLAST capacity of the $k$ th user is dominated by the weakest layer and it is given by [16]

$$
C_{k}^{\mathrm{ZF}-\mathrm{VBLAST}}=M_{\mathrm{T}} \min _{n=1,2, \ldots, M_{\mathrm{T}}}\left\{\log _{2}\left(1+\frac{\mathrm{SNR}}{M_{\mathrm{T}}\left\|W_{\mathrm{ZF}, n}^{k}\right\|^{2}}\right)\right\}
$$

where $W_{\mathrm{ZF}, n}^{k}$ is the $\mathrm{ZF}$ projection row for the $n$th layer of the $k$ th user and $M_{\mathrm{T}}$ is the number of layers (transmit antennas). Recall that the V-BLAST detector performs a series of interference nulling and cancellation operations [14]. At the $n$th stage, let $\mathbf{H}_{k, n}$ be the MIMO channel matrix for user $k$ after canceling the $n-1$ previous layers. The size of $\mathbf{H}_{k, n}$ is $M_{\mathrm{R}} \times M_{\mathrm{T}}-n+1$. Consequently, the ZF matrix at this stage is

$$
\mathbf{W}_{\mathrm{ZF}}^{k}=\left(\mathbf{H}_{k, n}^{H} \mathbf{H}_{k, n}\right)^{-1} \mathbf{H}_{k, n}^{H} \text {. }
$$

For optimal ordering, the V-BLAST detector starts with the strongest layer. At the $n$th stage, the strongest layer is the one with [15]

$$
\left\|W_{\mathrm{ZF}, n}^{k}\right\|^{2}=\min \left(\operatorname{diag}\left[\left(\mathbf{H}_{k, n}^{H} \mathbf{H}_{k, n}\right)^{-1}\right]\right)
$$

where diag $[\mathbf{A}]$ are the diagonal elements of $\mathbf{A}$. The corresponding post-processing SNR is [15]

$$
\mathrm{SNR}_{k, n}^{\mathrm{ZF}}=\frac{\mathrm{SNR}}{M_{\mathrm{T}}\left\|W_{\mathrm{ZF}, n}^{k}\right\|^{2}} .
$$

Therefore, the weakest layer that determines the capacity of V-BLAST is the one with the largest norm of the ZF projection row. Let $w_{k}=\max _{n=1,2, \ldots, M_{\mathrm{T}}}\left\|W_{\mathrm{ZF}, n}^{k}\right\|^{2}$ be the largest projection value for user $k$, then the scheduler that maximizes V-BLAST capacity will select the user with minimum $w_{k}$. In other words, the capacity maximization scheduling for V-BLAST selects the user with largest post-processing SNR of its weakest layer.
However, this criterion needs to perform ZF nulling and ordering to channel matrices of all users before selecting the best user, which requires a lot of computations. Therefore, we examine other suboptimal schedulers that are based on received MIMO channels before V-BLAST processing. The first one chooses the user with largest MIMO channel power $\left(\operatorname{trace}\left(\mathbf{H}_{k} \mathbf{H}_{k}^{H}\right)\right)$, and we refer to it as MaxSNR scheduler, which mimics the optimal scheduler for single-antenna systems [1]. The next scheduler measures the eigenspread of $\mathbf{H}_{k} \mathbf{H}_{k}^{H}$ and selects the user with the minimum eigenspread (MinES). The eigenspread is defined as

$$
s=\frac{\lambda_{\max }}{\lambda_{\min }}
$$

where $\lambda_{\max }$ and $\lambda_{\min }$ are the largest and smallest eigenvalues of $\mathbf{H}_{k} \mathbf{H}_{k}^{H}$, respectively. The eigenspread gives insight into the orthogonality of the channel. The smaller the value of $s$, the closer the matrix is to be orthogonal. The minimum value of $s$ is 1 , and it occurs when the channel matrix is orthogonal.

Let $\kappa$ be the condition number of $\mathbf{H}_{k}$. The eigenspread is related to $\kappa$ as

$$
\begin{aligned}
s & =\kappa^{2} \\
\text { where, } \kappa & =\frac{\rho_{\max }}{\rho_{\min }}
\end{aligned}
$$

and $\rho_{\max }$ and $\rho_{\min }$ are the largest and smallest singular values of $\mathbf{H}_{k}$. From this relation, we derive a scheduler that takes into account both the channel power and the eigenspread of $\mathbf{H}_{k}$. It selects the user that has the largest minimum singular value of $\mathbf{H}_{k}$. From (11), we have

$$
\begin{aligned}
& \frac{\rho_{\max }}{\rho_{\min }}=\sqrt{s} \\
& \rho_{\min }=\frac{\rho_{\max }}{\sqrt{s}} .
\end{aligned}
$$

Thus, selecting the largest $\rho_{\text {min }}$ means selecting a large $\rho_{\max }$, which measures the norm of $\mathbf{H}_{k}$ and hence the power, and a small eigenspread $(s)$. We will refer to this scheduler as MaxMinSV.

In addition, we compare the above user selection criteria with the product of eigenvalues (PEV) of $\mathbf{H}_{k} \mathbf{H}_{k}^{H}$, as proposed in [17] for multiuser downlink MIMO scheduling. We refer to this criterion as MaxPEV.

To summarize, the scheduling criteria considered for V-BLAST users are as follows:

- MaxVBLASTCapc: select the user with $\min _{k=1,2, \ldots, K}\left\{w_{k}\right\}$, where $w_{k}=\max _{n=1,2, \ldots, M_{\mathrm{T}}}$ $\left\{\left\|W_{\mathrm{ZF}, n}^{k}\right\|^{2}\right\}$ and $W_{\mathrm{ZF}, n}$ is defined in (8).

- MaxMinSV: select the user with maximum minimum singular value of $\mathbf{H}_{k}$.

- MaxPEV [17]: select the user with maximum product of eigenvalues of $\mathbf{H}_{k} \mathbf{H}_{k}^{H}$. 
- MinES: select the user with minimum eigenspread of $\mathbf{H}_{k} \mathbf{H}_{k}^{H}$ as defined in (10).

- MaxSNR [1]: select the user with maximum Frobenius norm of $\mathbf{H}_{k}\left(\operatorname{trace}\left(\mathbf{H}_{k} \mathbf{H}_{k}^{H}\right)\right)$.

- RR: round robin scheduling allows each user to transmit in a time division fashion.

\section{Capacity bounds on scheduling criteria}

In this section, we relate each of the scheduling criterion with the bounds on V-BLAST capacity. The best criterion technique is the one that is able to provide tighter bounds on

$$
\min _{k} \max _{n} \min _{i}\left\|W_{\mathrm{ZF}, n, i}^{k}\right\|^{2}
$$

where $\min _{i}\left\|W_{\mathrm{ZF}, n, i}^{k}\right\|^{2}$ is the minimum $i$ th diagonal value of $\left(\mathbf{H}_{k, n}^{H} \mathbf{H}_{k, n}\right)^{-1}$ of the $n$th layer of the $k$ th user. In this section, we will find bounds that are independent of $n$ and $i$ for each scheduling technique.

\section{MaxMinSV}

Choosing a user with maximum minimum singular value of $\mathbf{H}_{k}$ is the same as choosing a user with maximum minimum eigenvalue of $\mathbf{H}_{k} \mathbf{H}_{k}^{H}$. We have

$$
\min _{i}\left\|W_{\mathrm{ZF}, n, i}^{k}\right\|^{2}=\left[\left(\mathbf{H}_{k, n}^{H} \mathbf{H}_{k, n}\right)^{-1}\right]_{i, i} .
$$

This means that we are looking for the minimum $i$ th diagonal value of $\left(\mathbf{H}_{k, n}^{H} \mathbf{H}_{k, n}\right)^{-1}$. We can write it in the form of the following inequality:

$$
\begin{aligned}
\lambda_{\min }^{k, n}\left(\left(\mathbf{H}_{k, n}^{H} \mathbf{H}_{k, n}\right)^{-1}\right) & \leq \min _{i}\left\|W_{\mathrm{ZF}, n, i}^{k}\right\|^{2} \\
& \leq \lambda_{\max }^{k, n}\left(\left(\mathbf{H}_{k, n}^{H} \mathbf{H}_{k, n}\right)^{-1}\right) .
\end{aligned}
$$

Since $\mathbf{H}_{\mathbf{n}}{ }^{H} \mathbf{H}_{\mathbf{n}}$ is a squared Hermetian matrix, it can be written as

$$
\mathbf{H}_{k, n}^{H} \mathbf{H}_{k, n}=\mathbf{U} \Lambda_{k, n} \mathbf{U}^{H}
$$

where $\mathbf{U}$ is a unitary matrix with orthonormal eigenvectors and $\Lambda_{k, n}$ is the diagonal matrix of eigenvalues $\left[\lambda_{1}, \lambda_{2}, \ldots, \lambda_{M_{T}}\right]$. Another approach is to use QR decomposition as done in [18].

Using simple matrix manipulations, the inverse is written as

$$
\left[\mathbf{H}_{k, n}^{H} \mathbf{H}_{k, n}\right]^{-1}=\mathbf{U} \Lambda_{k, n}^{-1} \mathbf{U}^{H}
$$

The diagonal elements of $\Lambda_{k, n}^{-1}$ are $\left[\frac{1}{\lambda_{1}}, \frac{1}{\lambda_{2}}, \ldots, \frac{1}{\lambda_{M_{\mathrm{T}}}}\right]$. Therefore,

$$
\begin{aligned}
\lambda_{\min }^{k, n}\left(\left(\mathbf{H}_{k, n}^{H} \mathbf{H}_{k, n}\right)^{-1}\right) & =\frac{1}{\lambda_{\max }^{k, n}\left(\mathbf{H}_{k, n}^{H} \mathbf{H}_{k, n}\right)} \\
\lambda_{\max }^{k, n}\left(\left(\mathbf{H}_{k, n}^{H} \mathbf{H}_{k, n}\right)^{-1}\right) & =\frac{1}{\lambda_{\min }^{k, n}\left(\mathbf{H}_{k, n}^{H} \mathbf{H}_{k, n}\right)} .
\end{aligned}
$$

For the sake of simplicity, we will henceforth refer to $\lambda_{\max }^{k, n}\left(\mathbf{H}_{k, n}^{H} \mathbf{H}_{k, n}\right)$ and $\lambda_{\min }^{k, n}\left(\mathbf{H}_{k, n}^{H} \mathbf{H}_{k, n}\right)$ as $\lambda_{\max }^{k, n}$ and $\lambda_{\min }^{k, n}$, respectively. We now have

$$
\begin{aligned}
\frac{1}{\lambda_{\max }^{k, n}} & \leq \min _{i}\left\|W_{\mathrm{ZF}, n, i}^{k}\right\|^{2} \leq \frac{1}{\lambda_{\min }^{k, n}} \\
\frac{1}{\min _{n} \lambda_{\max }^{k, n}} & \leq \max _{n} \min _{i}\left\|W_{\mathrm{ZF}, n, i}^{k}\right\|^{2} \leq \frac{1}{\min _{n} \lambda_{\min }^{k, n}} .
\end{aligned}
$$

According to the inclusion principle for matrices, the minimum value of $\lambda_{\min }$ occurs at $n=1$ and the minimum vale of $\lambda_{\max }$ occurs at $n=M_{\mathrm{T}}$.

Now we have an upper bound given by

$$
\max _{n} \min _{i}\left\|W_{\mathrm{ZF}, n, i}^{k}\right\|^{2} \leq \frac{1}{\lambda_{\min }^{(k)}}
$$

or by taking the minimum over $K$ users

$$
\min _{k} \max _{n} \min _{i}\left\|W_{\mathrm{ZF}, n, i}^{k}\right\|^{2} \leq \frac{1}{\max _{k} \lambda_{\min }^{(k)}} .
$$

We will use this inequality to establish lower bounds on MaxMinSV.

For analytical tractability, we will focus on the case when $M_{\mathrm{T}}=M_{\mathrm{R}}$. The probability density function (pdf) of the smallest eigenvalue for the case of $M_{\mathrm{T}}=M_{\mathrm{R}}$ is given by [19]

$$
f_{\lambda_{\min }}(x)=M_{\mathrm{T}} e^{-x M_{\mathrm{T}}} u(x) .
$$

In this case, $\mu=\frac{1}{M_{\mathrm{T}}}$ and $\sigma=\frac{1}{M_{\mathrm{T}}}$, where $\mu$ and $\sigma^{2}$ represent the mean and variance. From chapter 10 of [20], we see that the maximum of $\lambda_{\min }$ will scale as $\frac{1}{M} \log K$. Therefore, we have a new bound on the capacity of VBLAST as

$$
C_{\mathrm{VBLAST}}^{\mathrm{ZF}} \geq M_{\mathrm{T}} \log _{2}\left(1+\frac{\mathrm{SNR} \log _{2} K}{M_{\mathrm{T}}^{2}}\right) .
$$

\section{MinES}

In this case, we start with the inequality

$$
\min _{i}\left\|W_{\mathrm{ZF}, n, i}^{k}\right\|^{2} \leq \frac{1}{\lambda_{\min }^{k, n}} \leq \kappa^{(k, n)} .
$$

Here $\kappa^{(k, n)}$ is the condition number of the matrix $\mathbf{H}_{k, n} \mathbf{H}_{k, n}^{H}$ defined as $\lambda_{\max } / \lambda_{\min }$. Now

$$
\max _{n} \min _{i}\left\|W_{\mathrm{ZF}, n, i}^{k}\right\|^{2} \leq \max _{n} \kappa^{(k, n)} .
$$


The condition number $\kappa$ is maximum when $n=1$, a lemma which follows from the inclusion principle of matrices. Therefore, we have

$$
\begin{aligned}
& \max _{n} \min _{i}\left\|W_{\mathrm{ZF}, n, i}^{k}\right\|^{2} \leq \kappa^{(k)} \\
& \min _{k} \max _{n} \min _{i}\left\|W_{\mathrm{ZF}, n, i}^{k}\right\|^{2} \leq \min _{k} \kappa^{(k)} .
\end{aligned}
$$

The pdf of $s$, the condition number of $\mathbf{H}_{k}$ for the case of $M_{\mathrm{T}}=M_{\mathrm{R}}$, is given by [19]

$$
f_{s / M_{\mathrm{T}}}(x)=\frac{8}{x^{3}} e^{-\frac{4}{x^{2}} u(x)} .
$$

As $\kappa=s^{2}$, we can show using a variable transformation argument that

$$
f_{\kappa}(x)=\frac{4 M_{\mathrm{T}}^{2}}{x^{2}} e^{-\frac{4 M_{\mathrm{T}}^{2}}{x}} u(x) .
$$

Now we find the upper bound on $\min _{k} \kappa^{(k)}$. Gordon et al. [21] provide bounds on inequalities for the minimum of random variables. These inequalities exist if a random variable $\xi$ satisfies the $(\alpha, \beta)$ condition defined as

$$
\begin{aligned}
& P(|\xi| \leq t) \leq \alpha t \\
& P(|\xi| \geq t) \leq e^{-\beta t}
\end{aligned}
$$

where $\alpha>0$ and $\beta>0$.

Then, the random variable $\xi$ is bounded as

$$
\frac{1}{2 \alpha K} \leq E\left[\min _{k} \xi^{(k)}\right] \leq \frac{1}{\beta K},
$$

where $E$ is the expected value.

Using the $\operatorname{pdf}$ of $\kappa$, we find that $\alpha=\beta=\frac{1}{4 M_{\mathrm{T}}^{2}}$. Now the upper bound on the minimum is given by

$$
E \min _{k} \xi^{(k)} \leq \frac{4 M_{\mathrm{T}}^{2}}{K}
$$

Now we have

$$
\min _{k} \max _{n} \min _{i}\left\|W_{\mathrm{ZF}, n, i}^{k}\right\|^{2} \leq \frac{4 M_{\mathrm{T}}^{2}}{K} .
$$

\section{MaxSNR}

We start with the established inequality

$$
\begin{aligned}
& \frac{1}{\max _{k} \min _{n} \operatorname{trace}\left(\mathbf{H}_{k, n} \mathbf{H}_{k, n}^{H}\right)} \\
\leq & \frac{1}{\max _{k} \min _{n} \lambda_{\max }^{k, n}} \leq \min _{k} \max _{n} \min _{i}\left\|W_{\mathrm{ZF}, n, i}^{k}\right\|^{2} .
\end{aligned}
$$

Again from the inclusion principle, the minimum of the trace occurs at $n=M_{\mathrm{T}}$.
Since we know the last layer, the pdf of trace $\left(\mathbf{H}_{k, n} \mathbf{H}_{k, n}^{H}\right)$ for the last $n$ becomes conditional. If we solve for the unordered VBLAST algorithm, we will get

$$
\frac{1}{\max _{k} \operatorname{trace}\left(\mathbf{h}_{k}^{H} \mathbf{h}_{k}\right)} \leq \min _{k} \max _{n} \min _{i}\left\|W_{\mathrm{ZF}, n, i}^{k}\right\|^{2}
$$

where $\mathbf{h}_{k}$ is a row vector containing $M_{\mathrm{R}}$ i.i.d complex Gaussian entries. In this case, the trace will be a chisquared random variable with $2 M_{\mathrm{R}}$ degrees of freedom. The pdf in this case is given as

$$
f_{t r}(x)=\frac{x^{M_{\mathrm{R}}-1}}{\left(M_{\mathrm{R}}-1\right) !} e^{-x} u(x)
$$

Now we use an upper bound on the maximum of the trace. Bertsimas et al. [22] provide us with a tight upper bound:

$$
E\left[\max _{k} \operatorname{trace}\left(\mathbf{h}_{k}^{H} \mathbf{h}_{k}\right)\right] \leq \mu+\sigma \sqrt{K-1}
$$

where $\mu$ and $\sigma^{2}$ are mean and variances, respectively. In this case, $\mu=2 M_{\mathrm{R}}$ and $\sigma^{2}=4 M_{\mathrm{R}}$. Now we have

$$
E\left[\max _{k} \operatorname{trace}\left(\mathbf{h}_{k}^{H} \mathbf{h}_{k}\right)\right] \leq 2 \sqrt{M_{\mathrm{R}}}\left(\sqrt{M_{\mathrm{R}}}+\sqrt{K-1}\right)
$$

Here we define an upper bound on capacity:

$C_{\mathrm{VBLAST}}^{\mathrm{ZF}-\text { unorder }} \leq M_{\mathrm{T}} \log _{2}\left(1+\frac{2 \mathrm{SNR}\left(\sqrt{M_{\mathrm{R}}}+\sqrt{K-1}\right)}{\sqrt{M_{\mathrm{T}}}}\right)$

\section{Simulation results BER performance}

Aggregate bit error rate (BER) performance of greedy scheduling over $4 \times 4$ multiuser MIMO systems is plotted in Figure 2 for 10 users and at $8 \mathrm{bps} / \mathrm{Hz}$ spectral efficiency. The MIMO channels are i.i.d complex Gaussian flat fading channels. The results show that the best criterion is the one that maximizes V-BLAST capacity (MaxVBLASTCapc). In addition, MaxMinSV, MaxPEV, and MinES schedulers capture most of the multiuser diversity, but MaxMinSV provides more gain since it takes into account the power of the MIMO channel. These criteria perform very close to MaxVBLASTCapc, which has more diversity at high SNR (sharper slope). The results in this figure also show that using maximum MIMO capacity as a scheduling criterion is not optimal for V-BLAST. The reason is the suboptimality of the V-BLAST detection algorithm.

For ORR scheduling, aggregate BER performance is shown in Figure 3. Unlike greedy scheduling, ORR 


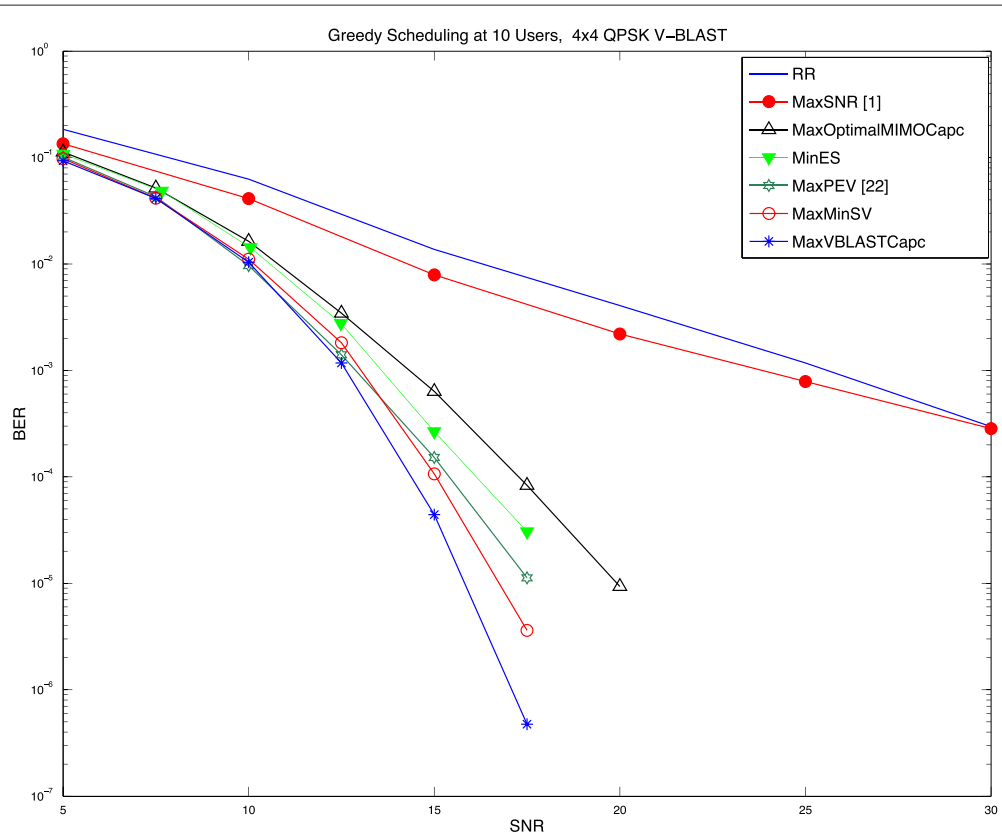

Figure 2 Aggregate BER for greedy scheduling for 10 users over $4 \times 4$ QPSK V-BLAST system.

scheduling criteria perform very close to each other and they gained around $12 \mathrm{~dB}$ compared to $\mathrm{RR}$ at $\mathrm{BER}=10^{-3}$. This suggests that for ORR scheduling, it is sufficient to use suboptimal less complex scheduling criteria such as MaxMinSV.

A comparison between greedy and ORR scheduling using MaxVBLASTCapc criterion is shown in Figure 4.
The ORR scheduler does not capture the whole multiuser diversity since it lies between the greedy algorithm and round robin. A loss in multiuser diversity is apparent from the slope of the BER curve. This is a trade-off that the system pays for achieving total fairness. However, the system still gains around $12 \mathrm{~dB}$ compared to the $\mathrm{RR}$ scheduler at 10 users. On the other hand, the greedy algorithm

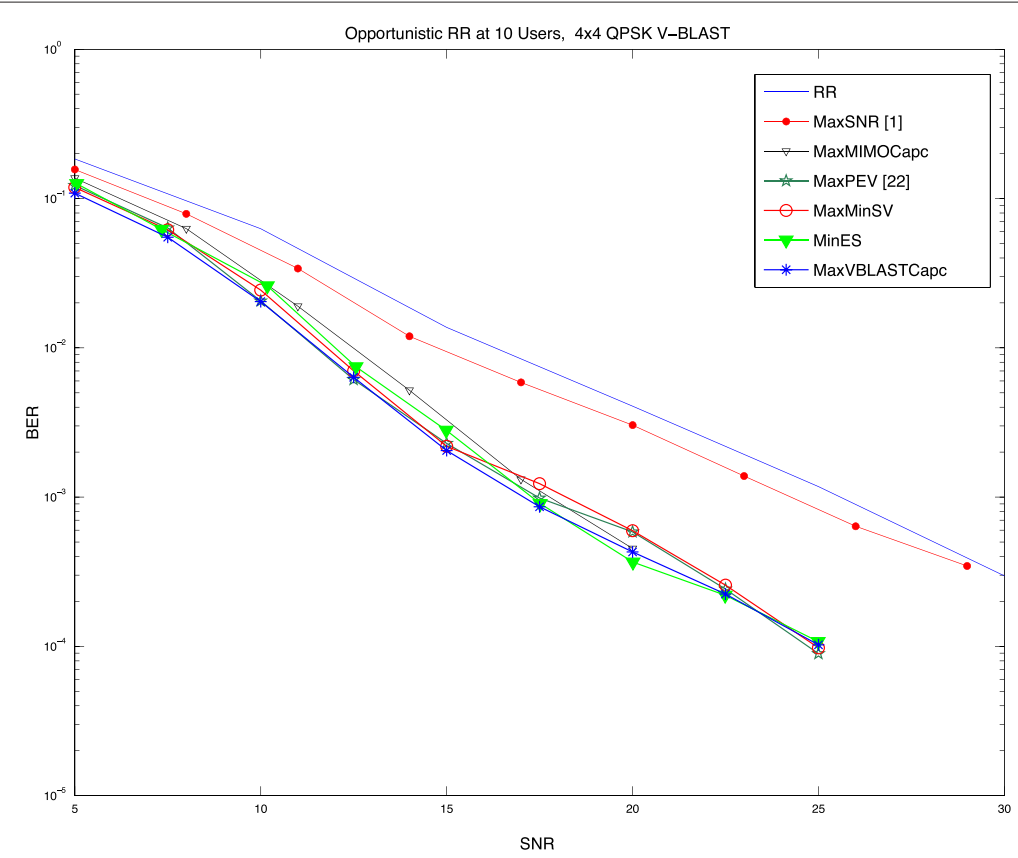

Figure 3 Aggregate BER for ORR scheduling for 10 users over $4 \times 4$ QPSK V-BLAST system. 


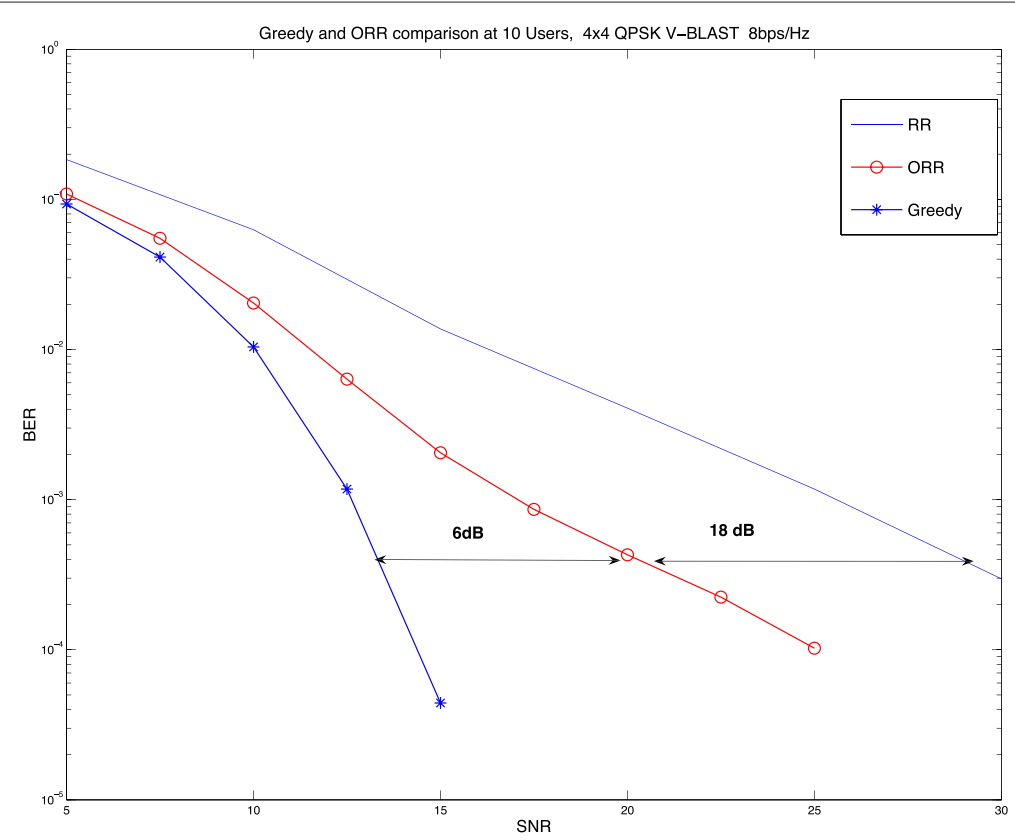

Figure 4 Greedy and ORR scheduling at 10 users and over $4 \times 4$ MIMO channels using MaxVBLASTCapc criterion.

performs much better than ORR, but it does not guarantee fairness for all users.

The scheduling gains of the ORR algorithm as a function of users are shown in Figure 5. This indicates that scheduling gains increase with increasing number of users, unlike the $R R$ algorithm, and that is a significant advantage for this scheduler.

\section{Effect of ZF and MMSE nulling matrices for V-BLAST} detection

As indicated before, V-BLAST could use ZF or MMSE to null out interfering layers. We performed a simulation study to compare the effect of ZF and MMSE nulling matrices used by V-BLAST. It is interesting to note that the SNR gain provided by MMSE in single-user systems

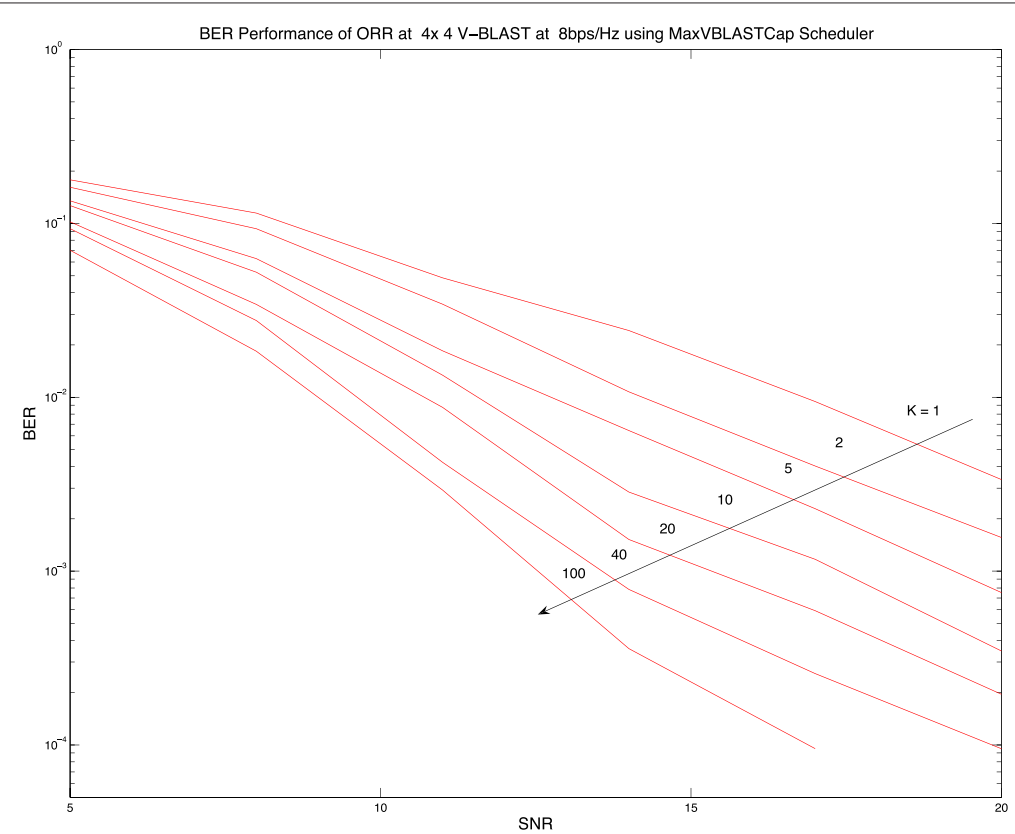

Figure 5 Effect of multiuser diversity of ORR scheduling algorithm over $4 \times \mathbf{4}$ MIMO channels. The number of users $K$ is set from 1 to 100 


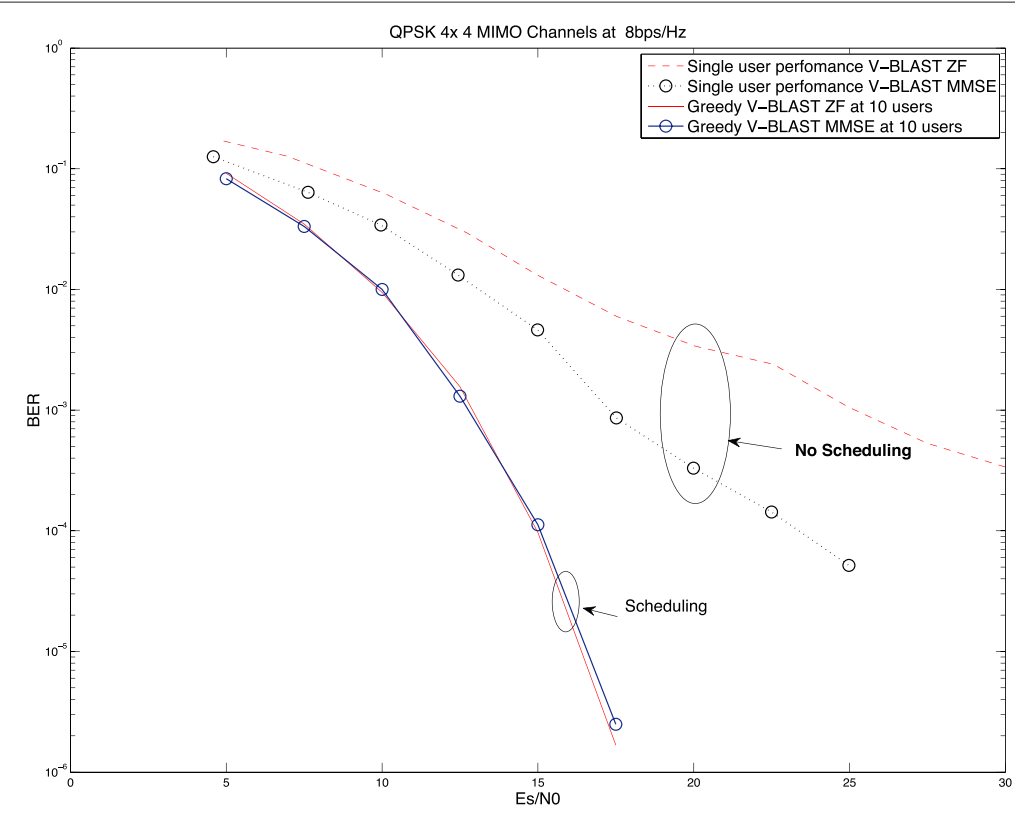

Figure 6 Effect of V-BLAST nulling matrix.

diminishes with multiuser diversity as shown in Figure 6. The result shows that there is an SNR gain for a single-user system. However, over multiuser systems with scheduling, this SNR gain diminishes because of multiuser diversity and the performance of ZF and MMSE is very close to each other.

\section{Outage capacity}

The capacity gains for ORR and greedy uplink MIMO scheduling at $10 \%$ outage versus the number of users are shown in Figures 7 and 8, respectively. Optimal MIMO capacity scheduling, as defined in (2), is estimated by assuming the availability of optimal MIMO modems. Therefore, it provides an upper bound for $\mathrm{V}$ BLAST scheduling algorithms. The MaxVBLASTCapc scheduler approaches optimal MIMO scheduling at a large number of users. For the ORR scheduler, the capacity gain is around $9 \mathrm{bps} / \mathrm{Hz}$ compared to RR scheduling at 100 users. In addition, system supported rates for MaxPEV, MaxMinSV, and MinES criteria are close to

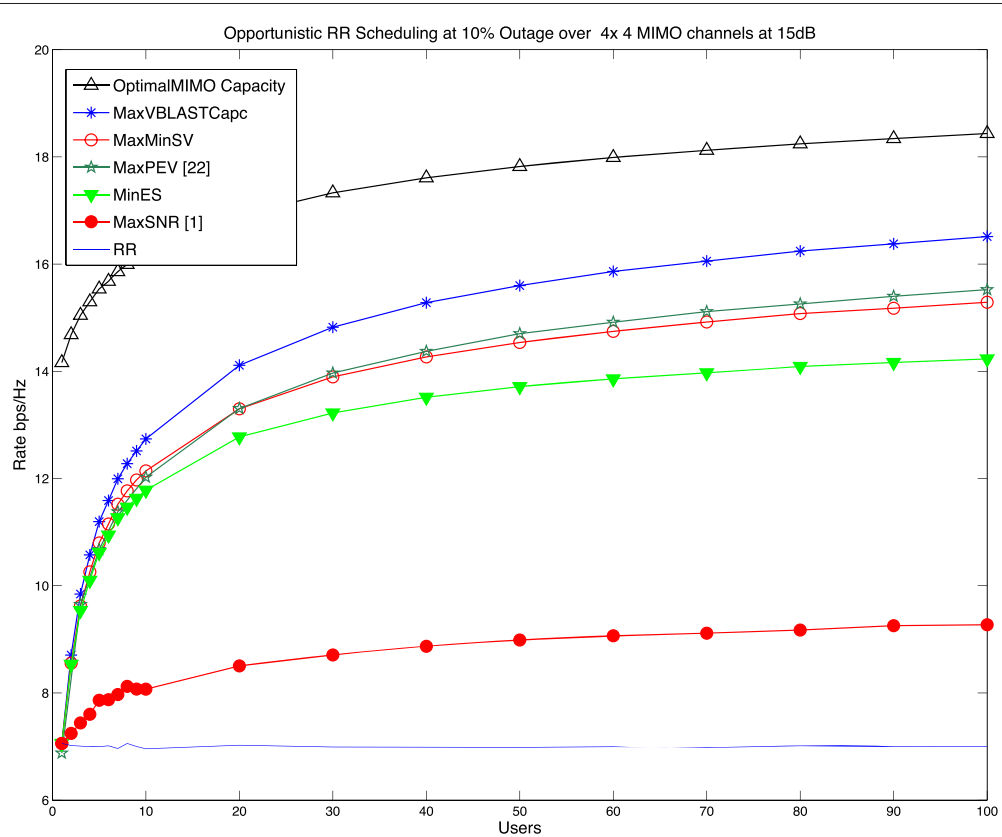

Figure $710 \%$ outage capacity of ORR scheduling versus number of users over $4 \times 4$ MIMO channels at $15 \mathrm{~dB}$. 


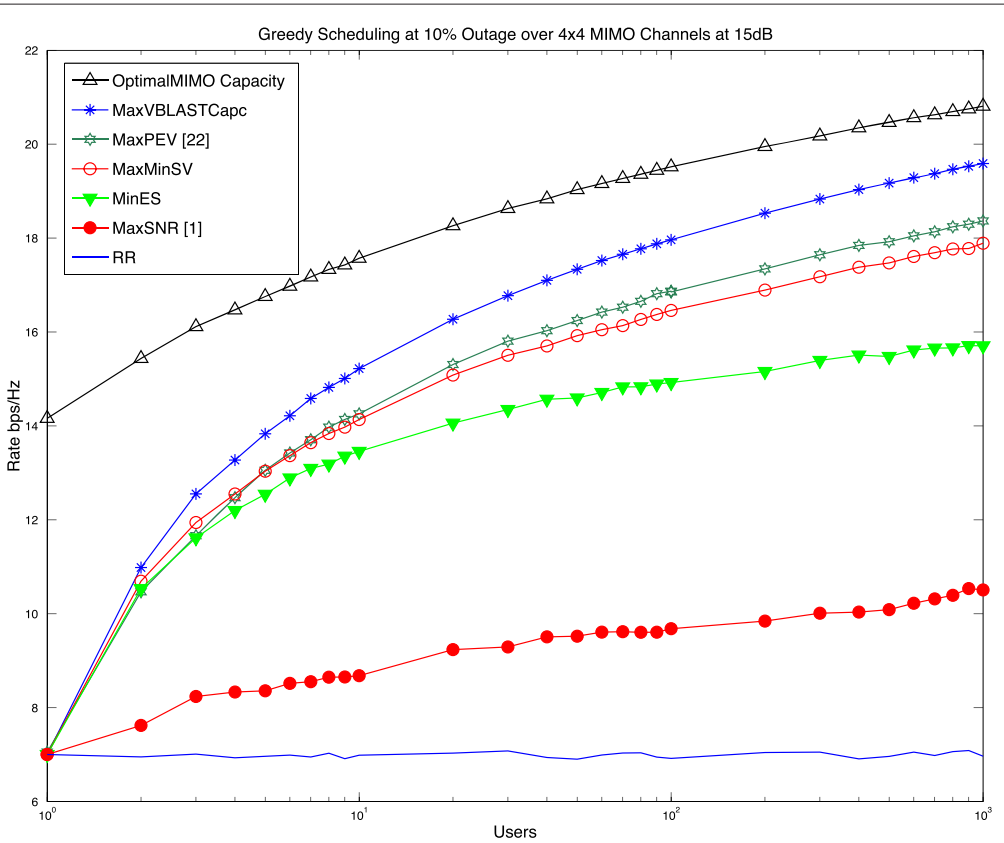

Figure $810 \%$ outage capacity of greedy scheduling versus number of users over $4 \times 4$ MIMO channels at $15 \mathrm{~dB}$.

MaxVBLASTCapc scheduling at a large number of users; the difference is within $2 \mathrm{bps} / \mathrm{Hz}$. These criteria greatly improve the information capacity of uplink systems at much less processing. The results in this figure also illustrate the poor performance of MaxSNR scheduling. It has very little gains even at large number of users. In addition, a capacity comparison of ORR and greedy algorithms is shown in Figure 9. The result shows 2 bps/Hz loss in capacity. This is a trade-off for the total fairness achieved by the ORR algorithm.

\section{Conclusions}

This paper proposed an opportunistic round robin (ORR) scheduling algorithm for uplink V-BLAST users over

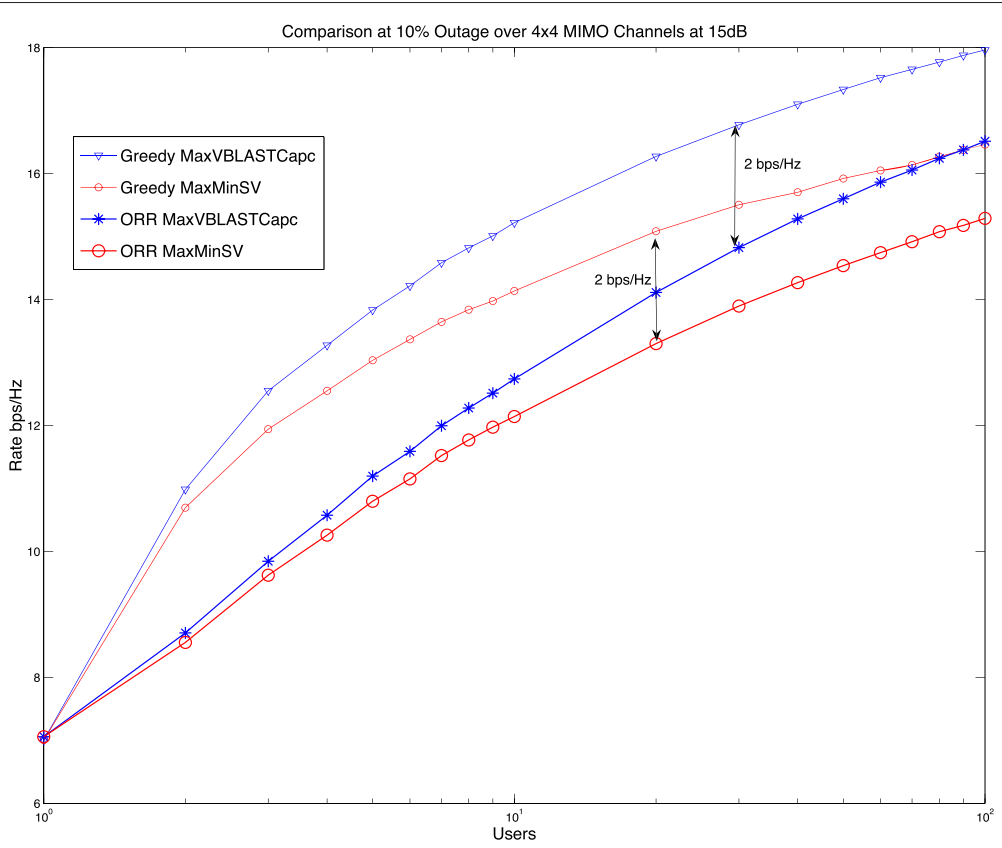

Figure $910 \%$ outage capacity comparison of greedy and ORR schedulers over $4 \times 4$ MIMO channels and at $15 \mathrm{~dB}$. 
multiuser MIMO channels. The proposed algorithm overcomes the drawbacks of greedy scheduling algorithm and provides full fairness to all users. In addition, error rate performance and outage capacities for user selection criteria were analyzed and compared for both ORR and greedy scheduling. User selection criteria for ORR perform very close to each other, while there are substantial differences in the greedy scheduling algorithm. This suggests that for ORR scheduling, it is sufficient to use suboptimal user selection criteria such as MaxMinSV.

The main results of this paper show the SNR and capacity gains obtained by ORR scheduling while providing full fairness to all users. Compared to round robin scheduling, the ORR scheduler provides around $12-\mathrm{dB}$ gain at $\mathrm{BER}=10^{-3}$ at 10 users. In addition, there is a fundamental trade-off between performance and fairness for ORR and greedy scheduling algorithms. The ORR scheduler does not capture the whole available multiuser diversity, but it provides full fairness to all users. On the other hand, greedy scheduling provides substantial performance improvements while there is no consideration for user fairness. Thus, ORR scheduling is an excellent candidate for next-generation high data rate system to satisfy certain quality of experience for all users.

\section{Competing interests}

The author has no competing interests.

\section{Acknowledgements}

The author would like to acknowledge the support provided by King Fahd University of Petroleum and Minerals (KFUPM) and King Abdulaziz City for Science and Technology (KACST) through the Science and Technology Unit at KFUPM for funding this work through project number 09-ELE781-4 as part of the National Science, Technology and Innovation Plan.

Received: 31 December 2013 Accepted: 13 July 2014

Published: 9 August 2014

\section{References}

1. R Knopp, P Humblet, Information capacity and power control in single cell multiuser communications, in Proc. IEEE Int. Computer Conf. (ICC'95) (Seattle, WA, 18-22 June 1995)

2. DNC Tse, Optimal power allocation over parallel Gaussian broadcast channels, in Proc. Int. Symp. Infrmation Theory (Ulm, Germany, 29 Jun - 4 July 1997)

3. RW Heath Jr, M Airy, AJ Paulraj, Multiuser diversity for MIMO wireless systems with linear receivers, in Thirty-Fifth Asilomar Conference on Signal, Systems and Computers, vol. 2 (IEEE, Pacific Grove, CA, USA, 4-7 Nov 2001), pp. 1194-1199

4. M Airy, S Shakkattai, RW Heath Jr, Spatially greedy scheduling in multi-user MIMO wireless systems, in Thirty-Seventh Asilomar Conference on Signals, Systems and Computers, vol. 1 (IEEE, Pacific Grove, CA, USA, 9-12 Nov 2003), pp. 982-986

5. L-C Wang, C-J Yeh, Scheduling for multiuser MIMO broadcast systems: transmit or receive beamforming? IEEE Wireless Commun. 9(9), 2779-2791 (2010)

6. C-H Pan, T-S Lee, Efficient QR-based multi-mode precoding for limited feedback multi-user MIMO systems. Wireless Personal Communications 74(2), 969-987 (2014)

7. R Gozali, RM Buehrer, BD Woerner, The impact of multiuser diversity on space-time block coding. IEEE Comm. Lett. 7(5), 213-215 (2003)

8. VKN Lau, Y Liu, TA Chen, Optimal multi-user space time scheduling for wireless communications. IEEE 56th VTC 2002-Fall. 4, 1939-1942 (2002)
9. S Al-Ghadhban, RM Buehrer, M Robert, Uplink scheduling criteria comparison for V-BLAST users, in 9th International Symposium on Signal Processing and Its Applications (Sharjah, UAE, 12-15 Feb 2007), pp. 1-4

10. J Choi, F Adachi, User selection criteria for multiuser systems with optimal and suboptimal LR based detectors. IEEE Trans. Signal Process. 58(10), 5463-5468 (2010)

11. L Bai, C Chen, J Choi, C Ling, Greedy user selection using a lattice reduction updating method for multiuser MIMO systems. IEEE Trans. Vehicular Technol. 60(1), 136-147 (2011)

12. J Mao, J Gao, Y Liu, G Xie, J Zhang, Robust multiuser MIMO scheduling algorithms with imperfect CSI. Sci. China Inf. Sci. 55(4), 815-826 (2012)

13. GJ Foschini, MJ Gan, On limits of wireless communications in a fading environment when using multiple antennas. Wireless Pers. Commun. 6, 311-335 (1998)

14. PW Wolniansky, GJ Foschini, GD Golden, RA Valenzuela, V-BLAST: an architecture for realizing very high data rates over the rich-scattering wireless channel, in Proc. ISSSE-98 (Pisa, Italy, 29 Sep - 2 Oct 1998), pp. 295-300

15. Y Jiang, MK Varanasi, J Li, Performance analysis of ZF and MMSE equalizers for MIMO systems: an in-depth study of the high SNR regime. IEEE Trans. Inf. Theory 57(4), 2008-2026 (2011)

16. CB Papadias, GJ Foschini, On the capacity of certain space-time coding schemes. EURASIP J. Appl. Signal Process. 5, 447-458 (2002)

17. L-N Tran, M Bengtsson, B Ottersten, Iterative precoder design and user scheduling for block-diagonalized systems. IEEE Trans. Signal Process. 60(7), 3726-3739 (2012)

18. H Lee, I Lee, Channel capacity of BLAST based on the zero forcing criterion. Vehicular Technol. Conf. VTC 2006-Spring IEEE 63rd. 4, 1615-1619 (2006)

19. A Edelman, Eigenvalues and condition numbers of random matrices. SIAM J. Matrix Anal. Appl. 9(4), 543-560 (1988)

20. HA David, Order Statistics, 1st edn. (Wiley, New York, 1970)

21. Y Gordon, AE Litvak, C Schutt, E Werner, On the minimum of several random variables. Proc. Am. Math. Soc. 134(12), 3669-3675 (2006)

22. D Bertsimas, K Natarajan, C-P Teo, Tight bounds on expected order statistics. Probability Eng. Informational Sci. 20(4), 667-686 (2006)

doi:10.1186/1687-1499-2014-128

Cite this article as: Al-Ghadhban: Opportunistic round robin scheduling for V-BLAST systems over multiuser MIMO channels. EURASIP Journal on Wireless Communications and Networking 2014 2014:128.

\section{Submit your manuscript to a SpringerOpen ${ }^{\circ}$ journal and benefit from:}

- Convenient online submission

- Rigorous peer review

- Immediate publication on acceptance

- Open access: articles freely available online

- High visibility within the field

- Retaining the copyright to your article

Submit your next manuscript at $>$ springeropen.com 